\title{
Vaccine campaign to target deadly childhood diarrhoea
}

\section{Programme to guard against second-biggest killer of under-fives rolls out across Africa.}

\section{BY DECLAN BUTLER}

$\mathrm{E}$ very year, more than one million children under the age of five die as a result of diarrhoea. It is the second-biggest killer in this age group, after pneumonia, and $40 \%$ of diarrhoea deaths are caused by rotavirus.

That horrific toll could soon fall, thanks to the first major roll-out of rotavirus vaccines in Africa, where half of rotavirus deaths occur. The programme was unveiled this week by the GAVI Alliance - formerly the Global Alliance for Vaccines and Immunisation - based in Geneva, Switzerland. The group hopes to immunize 50 million children against rotavirus in 40 of the world's poorest countries by 2015. Although rotavirus gastroenteritis is common worldwide, it kills very few children in richer countries, where hospitalization and intravenous rehydration are readily available, and where vaccination is becoming more common.

GAVI has already funded successful rotavirus-vaccination programmes in Nicaragua, Bolivia, Guyana and Honduras, but the effort is now ramping up to cover 13 countries in Africa and a further 4 elsewhere (see 'Rotavirus roll-out'). "It's an incredibly exciting time, to see this vaccine finally reaching the children in Africa," says John Wecker, head of vaccine access and delivery at the Program for Appropriate Technology in Health (PATH), an international organization based in Seattle, Washington, that runs the Rotavirus Vaccine Program (RVP) in partnership with the World Health Organization (WHO) and the US Centers for Disease Control and Prevention.

In the past it has often taken 15-20 years for a vaccine approved in rich countries to reach poorer ones, but GAVI funding has sharply reduced that lag for rotavirus and some other vaccines. The current rotavirus vaccines were first approved by the WHO and the US

\section{ONATURE.COM} Read more in Nature's vaccines special issue at: nature.com/vaccines
Food and Drug Administration just five years ago, on the basis of clinical trials in rich and middle-income countries. The RVP's trials of the vaccines in poorer countries were crucial for a wider roll-out, even though they showed that the vaccines are not quite as effective in those areas, where children are often malnourished and have other infectious diseases. The trials have proved, however, that the vaccines do reduce case numbers and, more importantly, substantially prevent serious disease and deaths. Unexpectedly, the vaccines also protect against multiple, fast-mutating strains of rotavirus, alleviating concerns among scientists that many different rotavirus vaccines might have been needed, with frequent reformulations.

Over the past few years, the RVP has sought to convince health officials in developing countries to push for vaccination, using evidence of the disease burden and the vaccine's effectiveness in their countries. It has helped to build disease-surveillance networks that will aid in identifying the places of greatest need, assessing the effectiveness of the vaccines and monitoring their safety.

Rotavirus-vaccine development suffered a setback in the 1990s, when RotaShield, made by Wyeth, had troublesome side effects. It was introduced in the United States in 1998, but was pulled from the market a few months later, after evidence arose that it elevated the risk of intussusception, an intestinal blockage than can prove fatal, by $1-2$ cases for every 10,000 vaccinated children.

Clinical trials of the newer Rotarix and RotaTeq vaccines, each comprising more than 60,000 children, found no elevated risk of intussusception. Later surveillance of the roll-out in Latin America, however, did show a risk, albeit far lower than that caused by RotaShield, as was reported in June (M M. Patel et al. N. Engl. J. Med. 364, 2283-2292; 2011). The WHO and national regulators who reviewed the data say that the benefits far outweigh the slightly elevated intussusception risk of $1-2$ in 100,000 vaccinated children, says Wecker.

GlaxoSmithKline, which makes Rotarix, has offered to make its vaccine available for the GAVI campaign at US $\$ 2.50$ per dose (vaccination requires two doses). That is $67 \%$ less than the current lowest price for the vaccine, although negotiations are still ongoing. Merck has promised to discount RotaTeq. GAVI expects prices to fall further as several manufacturers in emerging economies bring their own vaccines into production over the next few years.

The rotavirus effort follows the December 2010 launch of GAVI's vaccine programme targeting pneumococcus, a bacterium that causes pneumonia and other infections. This week, the alliance expanded that programme from 19 to 37 developing countries. Together, the campaigns are "definitely a game changer, no question”, says Wecker. "The vaccines will significantly reduce the burden of diarrhoea and pneumonia, the two leading killers of underfives in developing countries." - 